\title{
All-optical characterization of large-signal modulation bandwidth of a monolithically integrated DFB-EA
}

Blaaberg, Søren; Mulvad, Hans Christian Hansen; Oxenløwe, Leif Katsuo; Chacinski, Marek; Westergren, Urban; Stoltz, Björn

Published in:

Conference proceedings, CLEO/IQEC

Publication date:

2009

Document Version

Publisher's PDF, also known as Version of record

Link back to DTU Orbit

Citation (APA):

Blaaberg, S., Mulvad, H. C. H., Oxenløwe, L. K., Chacinski, M., Westergren, U., \& Stoltz, B. (2009). All-optical characterization of large-signal modulation bandwidth of a monolithically integrated DFB-EA. In Conference proceedings, CLEO/IQEC (pp. 1-2). IEEE.

\section{General rights}

Copyright and moral rights for the publications made accessible in the public portal are retained by the authors and/or other copyright owners and it is a condition of accessing publications that users recognise and abide by the legal requirements associated with these rights.

- Users may download and print one copy of any publication from the public portal for the purpose of private study or research.

- You may not further distribute the material or use it for any profit-making activity or commercial gain

- You may freely distribute the URL identifying the publication in the public portal 


\title{
All-optical characterization of large-signal modulation bandwidth of a monolithically integrated DFB-EA
}

\author{
S. Blaaberg ${ }^{1}$, H.C.H. Mulvad ${ }^{1}$, L.K. Oxenløwe ${ }^{1}$, M. Chacinski ${ }^{2}$, U. Westergren ${ }^{2}$ and B. Stoltz ${ }^{3}$ \\ 1: DTU Fotonik, Department of Photonics Engineering, Technical University of Denmark, DK-2800 Lyngby, Denmark \\ sblj@fotonik.dtu.dk \\ 2: Kista Photonic Research Center,Royal Institute of Technology,16440 Kista, Sweden, urban@kth.se \\ 3: Syntune AB, 16440 Kista, Sweden, bjorn.stoltz@syntune.com
}

\begin{abstract}
We use an all-optical method to characterize the modulation bandwidth of a DFB-EA designed for $100 \mathrm{~Gb} / \mathrm{s}$ Ethernet. In a large-signal wavelength conversion set-up, we show the device has an all-optical bandwidth of $83 \mathrm{GHz}$. (C)2009 Optical Society of America

OCIS codes: (250.7360) Waveguide modulators; (250.3140) Integrated optoelectronic curcuits;
\end{abstract}

\section{Introduction}

There is currently a strong trend towards adopting the so called Ethernet standard as the network protocol of the future [1]. $10 \mathrm{Gbit} / \mathrm{s}$ Ethernet $(10 \mathrm{GE})$ is the current standard but there are considerable efforts to push $10 \mathrm{GE}$ to 100 GE, by multiplexing in the electrical domain (electrical time division multiplexing, ETDM), as seen in e.g. [2] and [3-4]. Within the European EU-IST project, HECTO, a travelling-wave electroabsorption (EA) modulator is being developed to accommodate for $100 \mathrm{~Gb} / \mathrm{s}$ serial data modulation [5]. To characterize the bandwidth of such a device, special and expensive high-speed electronic equipment is usually required, equipment which is not easy to get hold of and is often limited to small-signal modulation.

In this paper, we use the all-optical method of wavelength conversion of $2 \mathrm{ps}$ wide pulses to measure the optical modulation bandwidth of a DFB-EA, an EA monolithically integrated with a DFB laser [5], in a large signal context. We demonstrate that we can convert the 2 ps control pulses into 4.3 ps DFB-EA pulses by cross-absorption modulation (XAM) in the EA section. This is the shortest pulse ever generated by XAM in an EA, to the best of our knowledge, and the Fourier spectrum reveals a 3-dB large-signal modulation bandwidth of $83 \mathrm{GHz}$.

\section{Experimental set-up}

The experimental set-up is depicted in Fig. 1. An optical sampling oscilloscope with a timing resolution of $0.9 \mathrm{ps}$ and an optical spectrum analyzer is used to measure the characteristic responses of the DFB-EA.

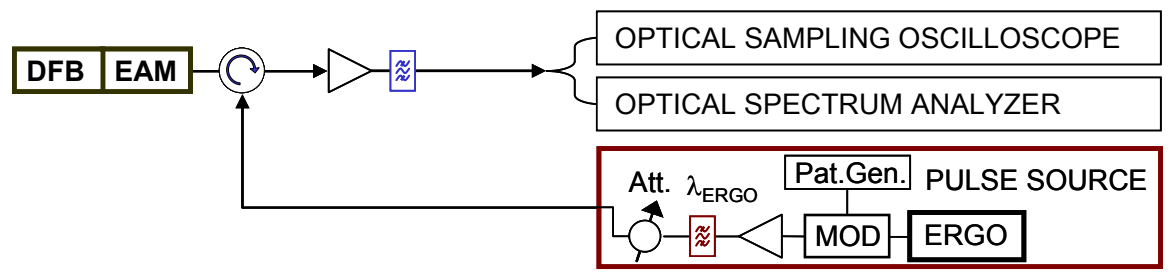

Fig. 1.Experimental set-up.

The control pulses are derived from an Erbium Glass pulse generating laser (ERGO), which produces $\sim 2$ ps pulses at $10 \mathrm{GHz}$. These pulses are then data modulated at $10 \mathrm{~Gb} / \mathrm{s}$ with various data patterns. For this characterization, a 1010 bit pattern is used. The pulses are amplified and a $3.5 \mathrm{~nm}$ filter reduces the ASE noise, and subsequently, the pulses are injected into the DFB-EA through an optical circulator. A variable attenuator (Att.) controls the power into the EAM. The DFB-EA is a multiple quantum well device grown in InGaAsP [5]. The active section is $180 \mu \mathrm{m}$ long, which is close to the spatial extent of the $2 \mathrm{ps}$ control pulse in this material. This means that the whole EA-section will be modulated by one full pulse. The numbers of QWs in the optical waveguide were 7 and 12 for the laser and modulator, respectively. Electrical isolation between the DFB and the modulator, as well as between active and passive segments of the modulator waveguide, was accomplished by careful design of the p-doped mesa structure and of the distance between the metal contacts [5]. This device is designed for electrical modulation and not for optical modulation, but still information on the intrinsic optical-only properties may be derived this way. The DFB laser is pumped at $55 \mathrm{~mA}$, which is approximately $40 \%$ above the threshold level. The lasing wavelength of the DFB laser is $1531.5 \mathrm{~nm}$. The reverse DC bias is supplied to the EA through small rf-probes connected through a $65 \mathrm{GHz}$ bias-T. The signal emitted by the DFB-EA, which includes both the wavelength converted part at $1531.5 \mathrm{~nm}$ and a reflected component at $1557 \mathrm{~nm}$, travels through the 


\section{CTuBB7.pdf}

circulator, an EDFA, and a bandpass filter (1.3 $\mathrm{nm}$ bandwidth) suppressing the light at $1557 \mathrm{~nm}$ before it is detected by an optical sampling oscilloscope and a optical spectrum analyzer, respectively. The DFB-EA chips are placed on a thermistor-equipped heat-sink, and operated at room temperature.

\section{Experimental results}

For the inspected device a low-frequency extinction ratio (ER) of $18 \mathrm{~dB}$ is measured at the output of the DFB-EA when modulating the DC bias applied to the EA between 0 and -5 Vat $1 \mathrm{kHz}$. The static transfer curves displayed in Fig. 2 show how much of this electrically induced absorption can be saturated by an optical CW control at $1557 \mathrm{~nm}$, for different DC-bias settings. Up to $14.9 \mathrm{~dB}$ extinction ratio is obtained this way. For higher input powers, the DFB laser starts to turn off. $14.9 \mathrm{~dB}$ is sufficient for many modulation applications.

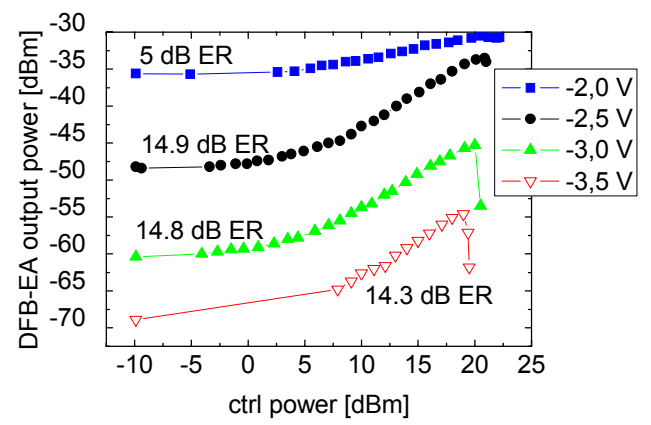

Fig.2. Static transfer curve: DFB-EA power as function of optical ctrl power (XAM).

Fig. 3 shows dynamic results. Fig. 3 (left) shows the XAM-generated pulses at the DFB laser wavelength as measured on the optical sampling oscilloscope with 0.9 ps timing resolution. Increasing the reverse bias from -3.1 to -3.7 volts reduces the pulse width by pulling in the carrier recovery tail. As short as 4.3 ps FWHM pulses are obtained. The contrast to the tail is about $15 \mathrm{~dB}$. Taking the Fourier transform of this $4.3 \mathrm{ps}$ pulse yields the spectrum in Fig. 3 (right). This spectrum reveals that the DFB-EA used for all-optical modulation has a 3-dB bandwidth of $83 \mathrm{GHz}$.
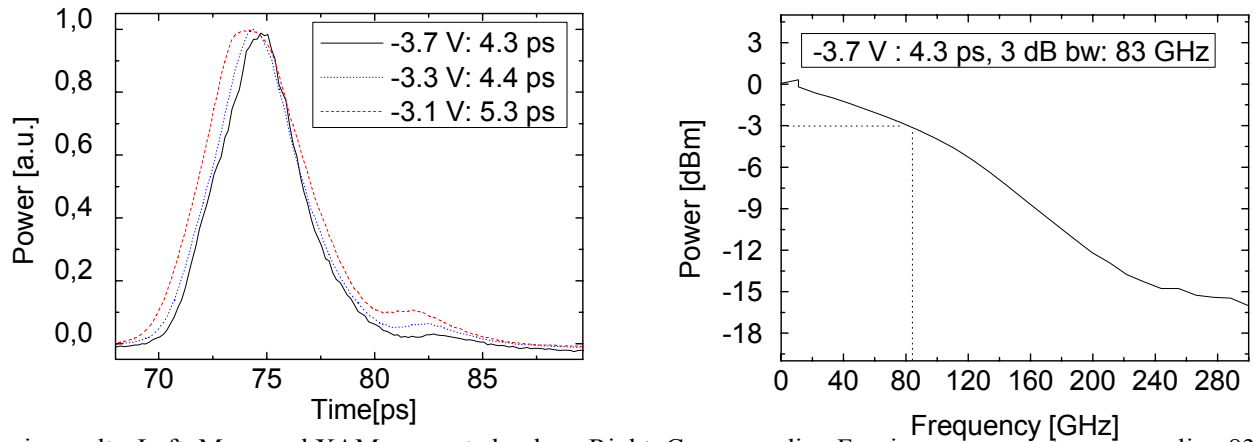

Fig. 3. Dynamic results. Left: Measured XAM-converted pulses. Right: Corresponding Fourier power spectrum, revealing $83 \mathrm{GHz}$ bandwidth.

\section{Conclusion}

This paper has characterised a DFB-EA designed for $100 \mathrm{~Gb} / \mathrm{s}$ Ethernet in an all-optical large-signal set-up. The DFB-EA has a static electro-optical extinction ratio of $18 \mathrm{~dB}$ and about $15 \mathrm{~dB}$ all-optical extinction ratio. Very short XAM-generated optical pulses can be generated with this component (4.3 ps FWHM), corresponding to a 3$\mathrm{dB}$ all-optical bandwidth of $83 \mathrm{GHz}$. This component is not designed for all-optical modulation, where a large amount of photo-induced carriers are generated and create field screening, so in an electro-optical approach it is expected that the bandwidth would be larger.

\section{Reference}

[1] IEEE 802.3 Working Group working on 100 GE standardisation, www.ieee802.org/3/

[2] G. Raybon and P.J. Winzer, " $100 \mathrm{~Gb} / \mathrm{s}$ challenges and solutions, OFC 2008, paper OTuG1, 2008

[3] GIBON homepage: http://www.ist-gibon.eu/

[4] HECTO homepage: http://www.hecto.eu/

[5] M. Chacinski, Urban Westergren, Björn Stoltz, and Lars Thylén, "Monolithically Integrated DFB-EA for $100 \mathrm{~Gb} / \mathrm{s}$ Ethernet", IEEE Electron Device Letters, Vol. 29, No. 12, December 2008 